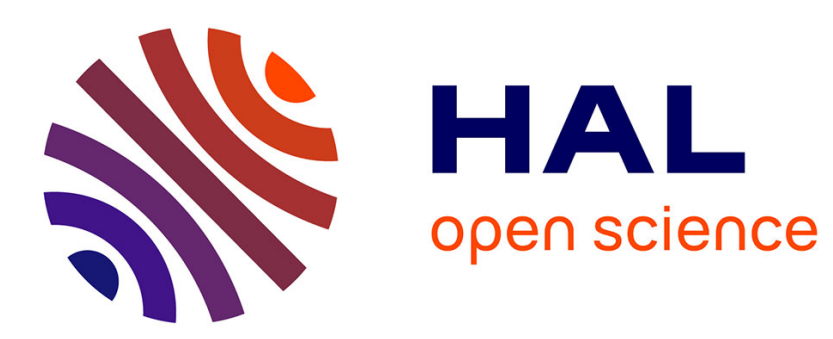

\title{
Serious Games for Home Based Rehabilitation: Inertial Sensor Energy Consumption
}

\author{
Halim Tannous, Dan Istrate, Aziz Benlarbi-Delai, Julien Sarrazin, \\ Marie-Christine Ho Ba Tho, Tien Tuan Dao
}

\section{- To cite this version:}

Halim Tannous, Dan Istrate, Aziz Benlarbi-Delai, Julien Sarrazin, Marie-Christine Ho Ba Tho, et al.. Serious Games for Home Based Rehabilitation: Inertial Sensor Energy Consumption. Innovation and Research in BioMedical engineering, 2018, 39 (6), pp.440-444. 10.1016/j.irbm.2018.10.014 . hal01915217

\section{HAL Id: hal-01915217 \\ https://hal.sorbonne-universite.fr/hal-01915217}

Submitted on 3 Sep 2019

HAL is a multi-disciplinary open access archive for the deposit and dissemination of scientific research documents, whether they are published or not. The documents may come from teaching and research institutions in France or abroad, or from public or private research centers.
L'archive ouverte pluridisciplinaire HAL, est destinée au dépôt et à la diffusion de documents scientifiques de niveau recherche, publiés ou non, émanant des établissements d'enseignement et de recherche français ou étrangers, des laboratoires publics ou privés. 
$\operatorname{IRBM} \bullet \bullet \bullet(\bullet \bullet \bullet \bullet) \bullet \bullet \bullet-\bullet \bullet \bullet$

JETSAN

\title{
Serious Games for Home Based Rehabilitation: Inertial Sensor Energy Consumption
}

\author{
H. Tannous ${ }^{\text {a }}$, D. Istrate ${ }^{\text {a }}$, A. Benlarbi-Delai ${ }^{b}$, J. Sarrazin ${ }^{b}$, M.-C. Ho Ba Tho ${ }^{\text {a }}$, T.T. Dao ${ }^{\text {a,* }}$ \\ a Sorbonne Université, Université de technologie de Compiègne, CNRS, UMR 7338 Biomechanics and Bioengineering (BMBI), Centre de recherche Royallieu - \\ CS 60319 - 60203 Compiègne cedex, France \\ b Sorbonne Université, UPMC Paris 06, UR2, L2E, F-75005, Paris, France
}

\section{H I G H L I G H T S}

- Battery life study of the inertial sensor.

- Current consumption study of the inertial sensor.

- Optimal configuration for a home based serious game system.

\section{A R T I C L E I N F O}

\section{Article history:}

Received 6 October 2017

Received in revised form 4 October 2018

Accepted 8 October 2018

Available online $\mathrm{xxxx}$

\section{Keywords:}

Energy consumption

Inertial sensors

Serious games

Home rehabilitation

\section{G R A P H I C A L A B S T R A C T}

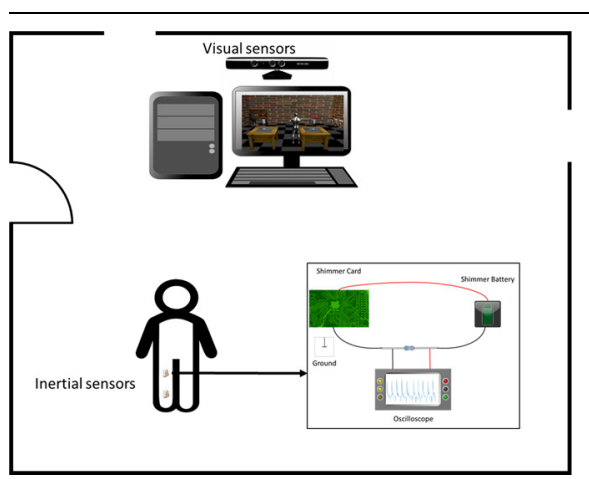

\begin{abstract}
A B S T R A C T
Background: Serious games have recently immerged as a good tool for physical rehabilitation. This new technology can be used at home, to complement a traditional, clinic based, rehabilitation program. To implement a serious game at home, we need to use multiple sensors to record patients' data. Many serious games use visual motion capture techniques, like the Kinect camera, due to their low price and high portability. On the other hand, some other systems use inertial sensors to collect data at a higher degree of accuracy. In previous works, we showed that a serious gaming system could benefit from combining data from different sensors. However, the use of inertial sensors, in a home-based setting, remains a challenge since they need to be supplied by an independent battery source, which could influence the acceptability of such systems.

Methods: In this paper, we present an energy consumption study, performed on the inertial sensors used in our serious game system.

Results: The results show that the sensors are rarely affected by environmental factors. They also show that the sensors can function continuously for about 14 hours without battery recharge.

Conclusion: Finally, these results allowed us to establish an optimal set up configuration for home based rehabilitation using serious games.
\end{abstract}

(C) 2018 AGBM. Published by Elsevier Masson SAS. All rights reserved.

\footnotetext{
* Corresponding author.

E-mail address: tien-tuan.dao@utc.fr (T.T. Dao).
}

\section{Introduction}

Physical rehabilitation is a long process that requires the intervention of a specific team of experts [1]. Usually, a patient undergoing this process will perform some sessions at the clinic, with 
expert supervision, and will be required later to do some exercises at home to remain active between clinical sessions. However, there are no current solutions for experts to monitor patient movements while performing exercises at home. In addition, patients drop home sessions due to the lack of motivation, and the high repetitiveness of these assigned exercises. Recently, these challenges have been the center of interest for many engineers and scientists, who used serious games as a complimentary tool for rehabilitation.

Researchers have implemented serious games for different types of pathologies. Parkinson's disease (PD) is one of these pathologies. Yu et al. developed a real-time Parkinson's rehabilitation environment using a visual motion capture system [2]. The system is implemented in a clinic and requires the patient to reach and step in different directions and speeds. A virtual avatar mimics the patient's movements on the screen. However, the system was never tested on PD patients. Paraskevopoulos et al. also studied serious games for PD rehabilitation [3]. They proposed a new design guideline for PD rehabilitation games, and developed two serious games. They tested these games on five PD patients. Stroke rehabilitation is another interesting pathology for serious games. Zannatha et al. developed a serious game using Kinect camera and EMG sensors [4]. The system has 4 games for upper limb rehabilitation, but was not tested on stroke patients. Some researchers were interested in general health and wellbeing of the elderly. Lozano-Quilis et al. developed an augmented reality system for multiple sclerosis using the Kinect camera [5]. The system has 3 different exercises, and was tested on 11 patients. The results showed that the patients accepted the system and felt safe while executing the exercises.

Implementing any system at home requires appropriate sensors to capture motion data. Two types of sensors are generally used for this purpose, visual motion capture sensors (e.g. Kinect) and inertial measurement units (IMU). The Kinect camera is widely used in serious game rehabilitation since it presents a portable solution with low cost $[4,5]$. However, Kinect has very low accuracy when used to estimate joint angles. Previous studies have shown that Kinect could estimate knee angle with an error of about $14.5^{\circ}$ [6-8]. This error is high when compared to the values accepted by medical experts to analyze joint data $\left(6^{\circ}\right.$ for higher extremities [9] and $5.5^{\circ}$ for lower extremities [10]). These problems can be avoided by using IMU sensors that are able to estimate angles at a higher degree of precision using complex mathematical filters [11,12]. Moreover, new studies have started investigating if a combination of different sensors could lead to higher precision in joint angle estimation. Atrsaei et al. studied a fusion algorithm using unscented Kalman [13]. The results showed that the new algorithm helped improving the position estimation of some upper body segments but not the angle estimation. Glonek et al. proposed an algorithm that averages inputs from Kinect and IMU sensors to estimate joint angles [14]. The study was validated with one subject performing multiple exercises. Other studies have tried to use multiple Kinect camera to capture human movements in a living lab [15]. In a previous work, we also proposed a new fusion algorithm between Kinect and IMU sensors, to better estimate the knee angle while performing a serious game rehabilitation exercise [6]. However, studies proposing the use of multiple types of sensors have not investigated how to use this technology in a home based environment to monitor patients or execute rehabilitation programs. The addition of wireless sensors and the necessity to recharge them between sessions could influence the user acceptability for these solutions. That is why we studied, in this paper, the battery and current consumption for the Shimmer3 IMU [16] that will be used with our previously developed serious game system [17-19].
The paper is organized as follows. Section 2 presents the studies performed, which includes the battery and current consumption study of the IMU sensors. Section 3 highlights the results of the battery consumption. Section 4 discusses the results. Finally, Section 5 concludes the study.

\section{Materials and methods}

\subsection{IMU study}

This test was performed on Shimmer3 IMU sensors [16], to identify how many sessions a patient can perform, without recharging the sensor. Our choice of sensor is based on our previous use of Shimmer3 in a data fusion study, between IMU sensors and Kinect camera [6]. In the study, Shimmer3 proved to be a very accurate sensor for joint angle estimation. The sensor contains a tri-axial accelerometer, gyroscope, and magnetometer that are always switched on during our tests. All these signals are needed to achieve a more accurate estimation of joint angle [6]. The study can be divided into two parts: battery life study and current consumption study.

\subsubsection{IMU battery life}

The battery life study includes 3 tests: 1 ) Effect of communication distance and sampling rate on battery life; 2) Effect of motion on battery life; and 3) Effect of multisensory streaming on battery life.

The sensors are charged until their batteries are full. Then, the sensors are connected to a developed application (using C\#) that saves their data to a file in real-time. The application allows streaming data from one to seven different sensors, using the Bluetooth communication protocol. The objective is to determine the effect of different conditions on battery life. Note that these tests were performed until battery depletion.

\subsubsection{IMU average current consumption}

The current consumption study includes 5 tests: 1) Current consumption until battery depletion at $51.2 \mathrm{~Hz} ; 2$ ) Effect of communication distance and sampling rate on current consumption; 3) Effect of motion on current consumption; 4) Effect of multisensory streaming on current consumption; and 5) Effect of placing sensor behind human body on current consumption.

For these tests, the sensor is taken out of its box, and the electronic chip is modified to allow the use of a multimeter (Fig. 1). Three trials were done for each test to ensure the reproducibility. The same application described above is used to connect the sensor to the PC. The multimeter is connected to the PC via a USB port, and an application allows us to save multimeter data to a file. The first test was performed to make sure that current consumption is homogeneous for a certain amount of time, which can allow us to record multiple trials continuously without recharging the sensor. For the other tests, the sensor(s) streamed for $10 \mathrm{~min}-$ utes and the current was collected from the multimeter. Note that the test that requires moving the sensor was done manually.

\subsubsection{IMU real-time current consumption}

This study aimed to provide a detailed description of the current pattern in real time, when the sensor is streaming to the PC. For this reason, we adopted the same scheme described in paragraph 2.2 , but we replaced the multimeter with a digital oscilloscope $(10 \mathrm{mV} / \mathrm{div})$. The current was recorded for 10 minutes at different streaming sampling rates. The delay between the received packets was also calculated to better understand the sending/reception mechanism put in place by the IMU manufacturers. 


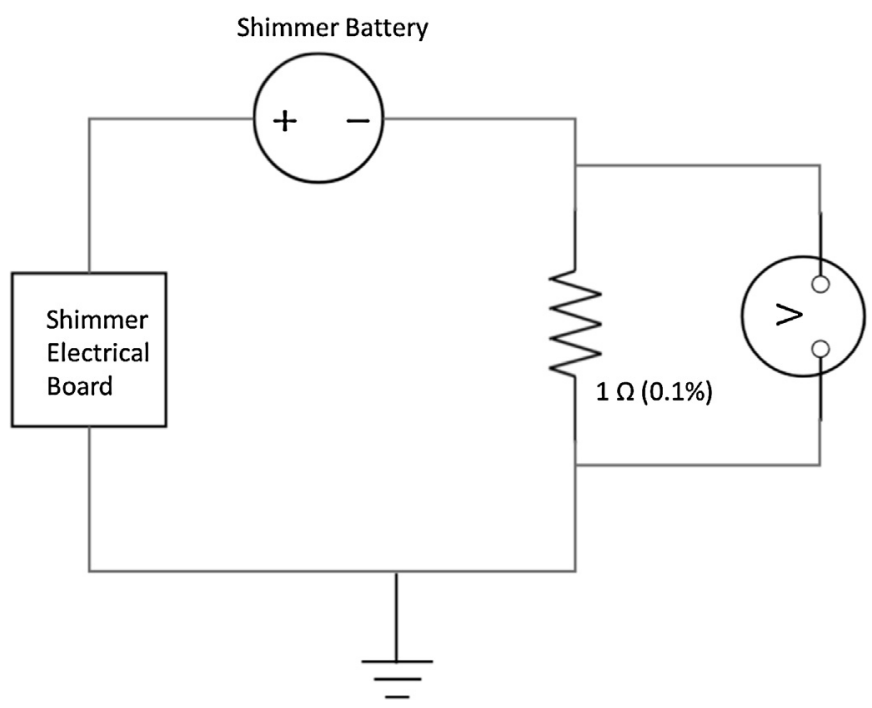

Fig. 1. Electric scheme for current measurement.

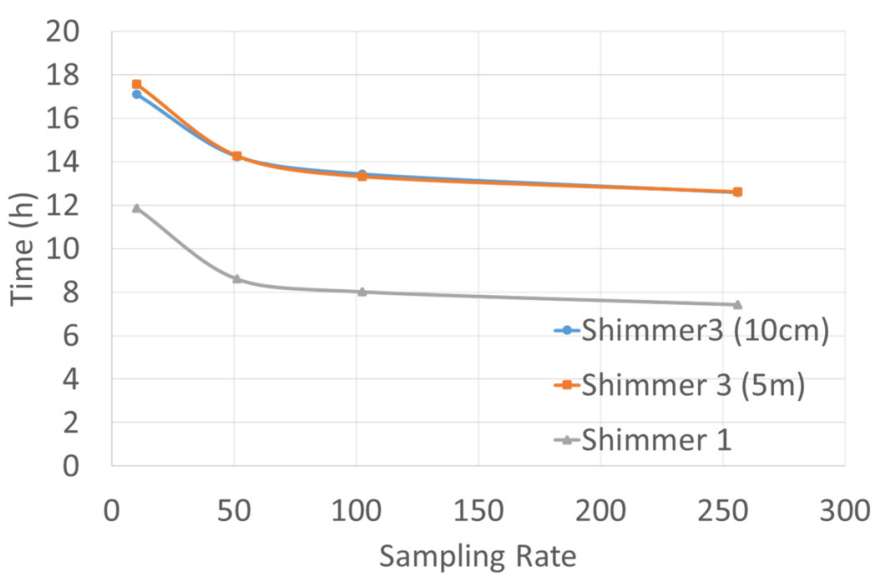

Fig. 2. Battery depletion time for different distances at different sampling rates.

\section{Results}

\subsection{Battery life}

Firstly, the effect of communication distance and sampling rate on battery life was studied. The results of this study are shown in Fig. 2. The results obtained with two different distances $(10 \mathrm{~cm}$ and $5 \mathrm{~m}$ ) from the PC were compared to results obtained in a previous study done on Shimmer1 [20].

The second study investigates the effect of motion on battery life. Since we needed to simulate motion for a long period of time, we attached the sensors to a small electric fan, and let it run overnight. The test was performed at $51.2 \mathrm{~Hz}$, as it is the sampling rate used in our previous studies to estimate body joint angles. This sampling rate proved to be sufficient enough to access the movement of the human body, which varies generally between $1-10 \mathrm{~Hz}$ in frequency [21]. The results show that when attaching the sensor to an electric fan overnight at $51.2 \mathrm{~Hz}$, the sensor streamed for $14.88 \mathrm{~h}$, compared to $14.9 \mathrm{~h}$ for a static sensor.

The third study shows the effect of multisensory streaming on battery life. The test was performed with 7 sensors versus 1 sensor, streaming continuously at $51.2 \mathrm{~Hz}$, until battery depletion. The results show that when connecting 7 sensors at the same time at $51.2 \mathrm{~Hz}$, they streamed for a mean battery depletion time of $14.71 \mathrm{~h}$ compared to $14.9 \mathrm{~h}$ when using one sensor.

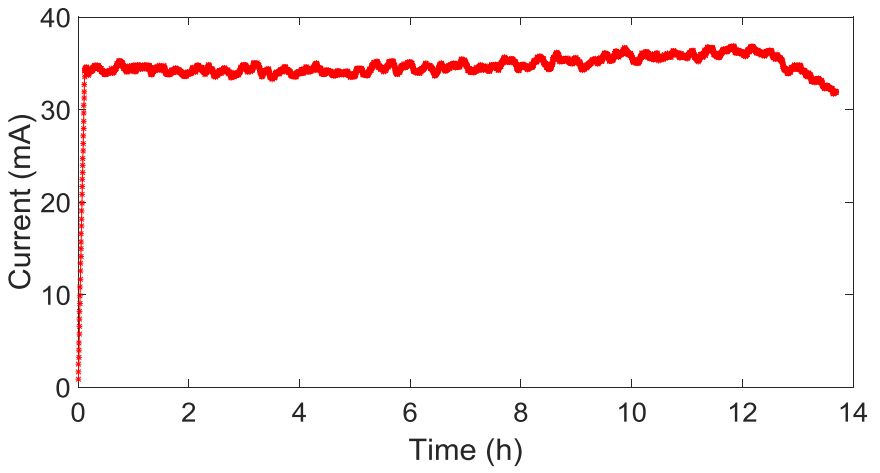

Fig. 3. Current dissipated during the test until battery depletion at $51.2 \mathrm{~Hz}$.

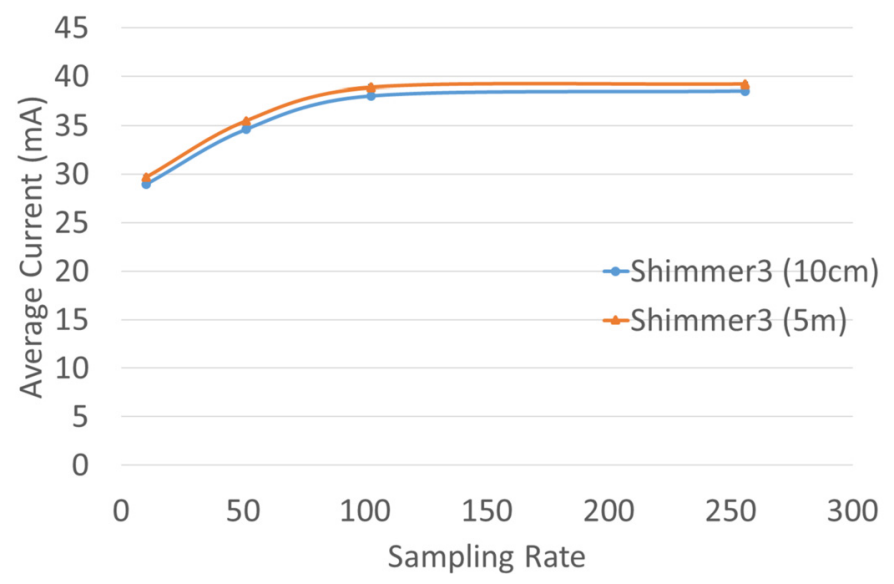

Fig. 4. Average current for different distances at different sampling rates.

\subsection{Average current consumption}

The first current consumption study investigated the changes in the average current consumed, for every $10 \mathrm{~min}$, in order to figure out if this average changes overtime. If the average is constant during a period of time, the measured current averages can be reliable, and we will avoid the need to recharge the sensor before each current consumption test. The result of this study is shown in Fig. 3.

The effect of the distance and sampling rate on average current consumption is shown in Fig. 4. The mean average current consumed presents the mean of 3 trials of 10 minutes each. The average is computed through computing the mean of the average 10 minute current consumed over 3 trials.

When moving the sensor for 10 minutes we obtained a mean average current equal to $34.8 \pm 4.46 \mathrm{~mA}$ versus $34.63 \pm 4.52 \mathrm{~mA}$ for a static sensor.

Multisensory streaming was studied for 2 different sampling rates $51.2 \mathrm{~Hz}$ (the sampling rate of interest) and $256 \mathrm{~Hz}$ (the highest sampling rate). Three trials were tested for each sampling rate. At $51.2 \mathrm{~Hz}$, when streaming 1 sensor we obtained a mean average current of $34.63 \pm 4.52 \mathrm{~mA}$ vs $34.48 \pm 2.41 \mathrm{~mA}$ for 7 sensors. At $256 \mathrm{~Hz}$, when streaming 1 sensor we obtained a mean average current of $38.55 \pm 4.49 \mathrm{~mA}$ vs $34.48 \pm 1.92 \mathrm{~mA}$ for 7 sensors. Finally, when placing the sensor behind a human body with respect to the remote central node, we obtained a mean average current of $35.14 \pm 4.62 \mathrm{~mA}$ vs $34.8 \pm 4.52 \mathrm{~mA}$ for a sensor placed next to the PC. 


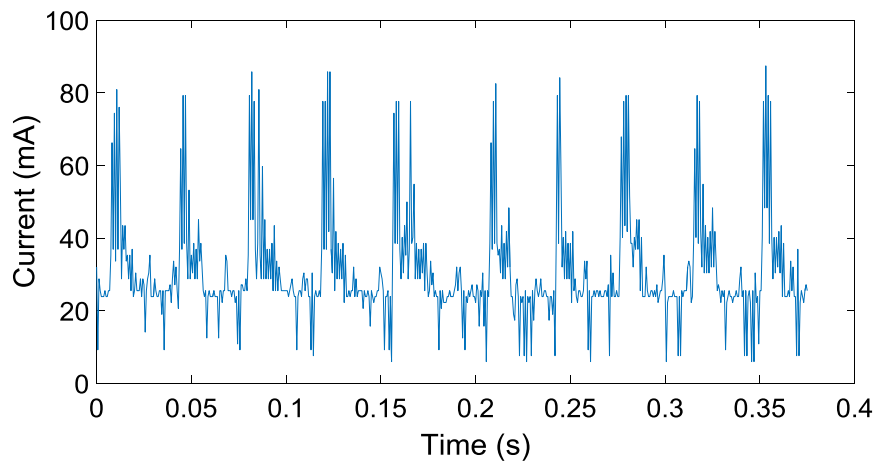

Fig. 5. Real-time dissipated current during streaming at $51.2 \mathrm{~Hz}$.

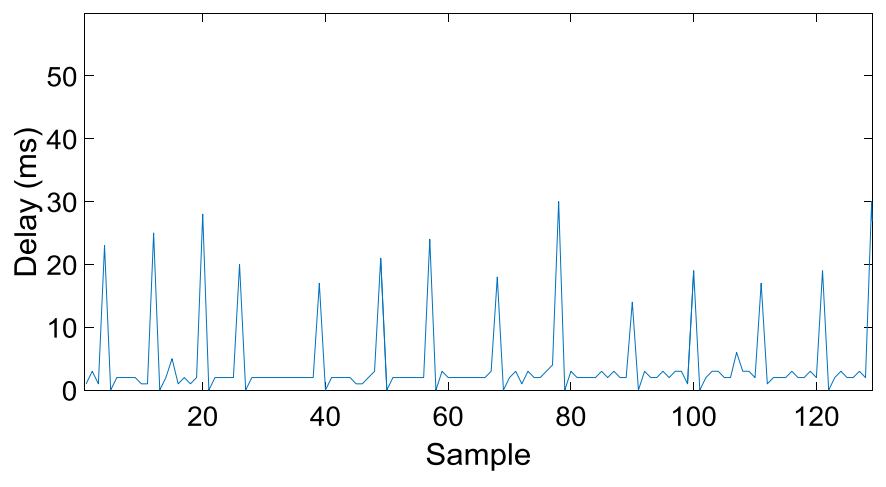

Fig. 6. Delay between samples received by the PC while streaming at $51.2 \mathrm{~Hz}$.

\subsection{Real-time current consumption}

The real-time current dissipated during streaming at $51.2 \mathrm{~Hz}$ was recorded using a digital oscilloscope. The results are presented in Fig. 5. The figure shows that there are periodically peaks of current consumption, and almost a constant current during the rest of the time. The period of these peaks is about $40 \mathrm{~ms}$. Other tests at different sampling rates showed no difference in these periods of peaks, and only a slight change in the average constant current consumed. We also measured the delays between samples received by the PC, presented in Fig. 6.

\section{Discussion}

The battery life and current consumption study shows that the usage conditions rarely affect Shimmer3 sensor efficiency. The first battery life test showed that the distance and the sampling rate do not affect battery life. Fig. 2 shows that Shimmer1 battery life is lower than that of Shimmer3 but varies in the same manner. Shimmer 1.0 does not contain a magnetometer, and its battery has a capacity of $280 \mathrm{mAh}$ versus $450 \mathrm{mAh}$ in Shimmer3, which could explain these results. The second battery life test, concerning sensor movement showed no significant difference in battery life between a static and a dynamic sensor. The same observation was noted when comparing multisensory streaming versus single sensor streaming.

The current consumption seemed homogeneous for the first $8 \mathrm{~h}$ (Fig. 3) when we performed a test until battery depletion. This means that we can test current consumption without recharging the sensor after each trial. The average current seems to be slightly affected by distance (Fig. 4). However, when it comes to sensor movement, multisensory streaming and on body streaming, the current consumption does not exhibit significant changes. These results show that Shimmer3 sensor is not affected by envi-
Table 1

Optimal conditions for home based rehabilitation using serious games.

\begin{tabular}{|c|c|c|}
\hline Criteria & Recommendations & Purposes \\
\hline Distance from the system & At least 2 meters & $\begin{array}{l}\text { Optimal condition for } \\
\text { Kinect joint estimation }\end{array}$ \\
\hline Room dimensions & At least $4 \times 4$ meters & $\begin{array}{l}\text { Patient movement in } \\
\text { spacious area }\end{array}$ \\
\hline Room lighting & Well-lit room & $\begin{array}{l}\text { Optimal condition for } \\
\text { Kinect joint estimation }\end{array}$ \\
\hline Clothe type & $\begin{array}{l}\text { Shorts and t-shirts } \\
\text { with well-fitting size }\end{array}$ & $\begin{array}{l}\text { Maximal contact between } \\
\text { inertial sensor and the } \\
\text { body }\end{array}$ \\
\hline Number of sensors & $\begin{array}{l}\text { A maximum of } 7 \\
\text { sensors }\end{array}$ & $\begin{array}{l}\text { Maximal number in a } \\
\text { Bluetooth piconet }\end{array}$ \\
\hline Sensor battery & $\begin{array}{l}\text { Recharge after } 25 \\
\text { sessions }\end{array}$ & $\begin{array}{l}\text { Battery depletion after } 14 \\
\text { hours of usage }\end{array}$ \\
\hline
\end{tabular}

ronmental factors. The only constraint of the system is a maximum number of 7 sensors.

The third study was to investigate the real-time current dissipated during streaming. The Fig. 5 shows that there are peaks of current consumption at times. Since the frequency of current consumption peaks did not change with different sampling rates, we proposed a hypothesis that the sensor saves the samples recorded at a particular sampling rate, and then allocates periods of $20 \mathrm{~ms}$ to send all the saved data. Thus, with higher sampling rates, there is no increase in the number of peaks, but an increase in the mean current dissipated, which causes the battery to deplete much faster. To confirm this hypothesis, we measured the delay between the received samples by the PC, presented in Fig. 6. The results of this study confirmed our hypothesis, as there are some samples that are received with big delays. These delays happen when the sensor sends a sample at the end of a sending window, and then sends the next one in the next sending window. After these peaks, the delays get smaller as the sensor sends the samples continuously.

Therefore based on these results, and optimal conditions proposed by the Kinect manufacturers, we can propose an optimal configuration for a home based serious game system (Table 1). We recommend a minimal distance of 2 meters between the user and the visual sensor, to obtain an optimal joint recognition through the Kinect body joint estimation, this requires a room of at least $4 \times 4$ meters, where the furniture is not allowed between the player and the sensors. Moreover, the visual sensor works in an optimal condition when the room is well lit. When it comes to inertial sensors, the type of clothes worn by the user affects them generally. That is why we recommend that the users wears shorts and t-shirts, or clothes that are not larger than their size, in order to maximize the contact between the sensor and the body joint. These recommendations are presented in Table 1.

\section{Conclusion}

In this paper, we presented a study of the energy consumption of Shimmer3 inertial sensors. This technical study shows that shimmer sensors can hold up to 14 hours when streaming continuously at $51.2 \mathrm{~Hz}$. The study also investigated how the current is consumed in real-time, which could help us understand how to optimize the use of these sensors. In particular, this study showed that Shimmer3 sensors are not affected by environmental factors, and thus can be used without any limiting conditions. The user should only charge the battery once after about 25 rehabilitation sessions of 30 mins each. Moreover, our system that uses the Kinect camera combined with inertial sensors has to take into account the limitations of the camera alone. Finally, we gave some recommendations on the optimal room and user conditions to deploy serious games at home. In future works, we will 
study the dynamic joint behavior to detect false movements, and generate feedbacks to help patients enhance their rehabilitation performance, and achieve our complete, real-time, home based rehabilitation system [22].

\section{Human and animal rights}

The authors declare that there are no issues related to human and animal rights in this present work.

\section{Conflict of interest}

The authors declare that there is no conflict of interest related to this present work.

\section{Acknowledgements}

This work was funded within the framework of EBIOMED Chair-IUIS (Institut Universitaire d'Ingénierie en Santé). This work was carried out in the framework of the Labex MS2T, which is funded by the French Government, through the program "Investments for the future" managed by the National Agency for Research (Reference ANR-11-IDEX-0004-02). This work is also a part of the STIC AMSUD - EMONITOR project.

\section{References}

[1] www.urmc.rochester.edu/encyclopedia/ [Last accessed: September 2017].

[2] Yu W, Vuong C, Ingalls T. An interactive multimedia system for Parkinson's patient rehabilitation. In: Virtual and mixed reality - systems and applications, part II, HCI 2011; 2011. p. 129-37.

[3] Paraskevopoulos IT, Tsekleves E, Craig C, Whyatt C, Cosmas J. Design guidelines for developing customised serious games for Parkinson's disease rehabilitation using bespoke game sensors. Comput Entertain 2014;5:413-24. https://doi.org/ 10.1016/j.entcom.2014.10.006.

[4] Ibarra Zannatha JM, Tamayo AJM, Sánchez ADG, Delgado JEL, Cheu LER, Arévalo WAS. Development of a system based on 3D vision, interactive virtual environments, ergonometric signals and a humanoid for stroke rehabilitation. Comput Methods Programs Biomed 2013;112:239-49. https://doi.org/10.1016/ j.cmpb.2013.04.021.

[5] Lozano-Quilis J-A, Gil-Gómez H, Gil-Gómez J-A, Albiol-Pérez S, PalaciosNavarro G, Fardoun HM, et al. Virtual rehabilitation for multiple sclerosis using a kinect-based system: randomized controlled trial. JMIR Serious Games 2014;2:e12. https://doi.org/10.2196/games.2933.

[6] Tannous H, Istrate D, Benlarbi-Delai A, Sarrazin J, Gamet D, Ho Ba Tho MC, et al. A new multi-sensor fusion scheme to improve the accuracy of knee flexion kinematics for functional rehabilitation movements. Sensors (Switzerland) 2016:16. https://doi.org/10.3390/s16111914.
[7] Plantard P, Auvinet E, Le Pierres A-S, Multon F. Pose estimation with a Kinect for ergonomic studies: evaluation of the accuracy using a virtual mannequin. Sensors (Switzerland) 2015;15:1785-803. https://doi.org/10.3390/s150101785.

[8] Bonnechère B, Jansen B, Salvia P, Bouzahouene H, Omelina L, Moiseev F, et al. Validity and reliability of the Kinect within functional assessment activities: comparison with standard stereophotogrammetry. Gait Posture 2014;39:593-8. https://doi.org/10.1016/j.gaitpost.2013.09.018.

[9] Gajdosik RL, Bohannon RW. Goniometry emphasizing reliability and validity clinical measurement of range of motion: review of clinical measurement of range of motion review of goniometry emphasizing reliability and validity. Phys Ther 1987;67:1867-72.

[10] Brosseau L, Tousignant M, Budd J, Chartier N, Duciaume L, Plamondon S, et al. Intratester and intertester reliability and criterion validity of the parallelogram and universal goniometers for active knee flexion in healthy subjects. Physiother Res Int 1997;2:150-66. https://doi.org/10.1002/pri.97.

[11] Madgwick SOH, Harrison AJL, Vaidyanathan A. Estimation of IMU and MARG orientation using a gradient descent algorithm. In: IEEE international conference on rehabilitation robotics: [proceedings] 2011; 2011:5975346.

[12] Marins JL, Bachmann ER, McGhee RB, Zyda MJ. An extended Kalman filter for quaternion-based orientation estimation using MARG sensors. In: Proceedings 2001 IEEE/RSJ international conference on intelligent robots and systems expanding the societal role of robotics in the the next millennium (Cat No01CH37180), vol. 4. 2003. p. 2003-11.

[13] Atrsaei A, Salarieh H, Alasty A. Human arm motion tracking by orientationbased fusion of inertial sensors and kinect using unscented Kalman filter. J Biomech Eng 2016;138:91005. https://doi.org/10.1115/1.4034170.

[14] Glonek G, Wojciechowski A. Hybrid method of human limb joints positioninghand movement case study. In: Information technologies in medicine; 2016. p. 307-20.

[15] Sevrin L, Noury N, Abouchi N, Jumel F, Massot B, Saraydaryan J. Preliminary results on algorithms for multi-kinect trajectory fusion in a living lab. IRBM 2015;36:361-6. https://doi.org/10.1016/j.irbm.2015.10.003.

[16] www.shimmersensing.com/ [Last accessed: September, 2017].

[17] Tannous H, Istrate D, Ho Ba Tho MC, Dao TT. Serious game and functional rehabilitation for the lower limbs [Jeux sérieux et rééducation fonctionnelle des membres inférieurs]. Eur Res Telemed 2016;5:65-9. https://doi.org/10.1016/j. eurtel.2016.05.001.

[18] Tannous H, Istrate D, Ho Ba Tho MC, Dao TT. Serious game and functional rehabilitation for the lower limbs. Eur Res Telemed / Rech Eur Téléméd 2016;5:65-9. https://doi.org/10.1016/j.eurtel.2016.05.001.

[19] Idriss M, Tannous H, Istrate D, Perrochon A, Salle J-Y, Ho Ba Tho MC, et al. Rehabilitation-oriented serious game development and evaluation guidelines for musculoskeletal disorders. JMIR Serious Games 2017:5:e14. https://doi.org/ 10.2196/games.7284.

[20] Burns A, Greene BR, McGrath MJ, O’Shea TJ, Kuris B, Ayer SM, et al. SHIMMERTM - a wireless sensor platform for noninvasive biomedical research. IEEE Sens J 2010;10:1527-34. https://doi.org/10.1109/JSEN.2010.2045498.

[21] Zeng H, Zhao Y. Sensing movement: microsensors for body motion measurement. Sensors 2011;11:638-60. https://doi.org/10.3390/s110100638.

[22] Dao TT, Tannous H, Pouletaut P, Gamet D, Istrate D, Ho Ba Tho MC. Interactive and connected rehabilitation systems for E-health. IRBM 2016;37:289-96. https://doi.org/10.1016/j.irbm.2016.02.003. 